\title{
El trabajo en equipo como parte de un sistema de salud
}

\author{
Teamwork as part of a health system \\ Federico L Rodríguez Weber, * Ricardo Secín Diep, ${ }^{\ddagger}$ José Luis Ramírez Arias ${ }^{\S}$ \\ Citar como: Rodríguez WFL, Secín DR, Ramírez AJL. El trabajo en equipo como parte de un sistema \\ de salud. Acta Med GA. 2021; 19 (4): 477-479. https://dx.doi.org/10.35366/102530
}

En la unión está la fuerza, la alianza conlleva el éxito, estas frases entre otras se utilizan con frecuencia para inferir la importancia del trabajo en equipo.

Al trabajar en equipo se suman fuerzas, actitudes, aptitudes y conocimientos de los diferentes integrantes de una fuerza de trabajo, tienen como fin potenciar entre todos los resultados, la eficiencia y la eficacia de una empresa, lo que obliga a cada quien a responsabilizarse de las tareas asignadas, para ello debe haber empatía, armonía, colaboración y acuerdo en toma de decisiones, y todo lo anterior conduce a que exista un líder que coordine las acciones dando confianza, orientación, apoyo, distribuya las cargas de trabajo y que además genere ánimo en el grupo, permitiendo que éste sea autosuficiente, creativo y con capacidad de respuesta a cambios eventuales. Es muy importante que el líder tenga capacidad de evaluar el trabajo de cada uno de los integrantes de la fuerza de trabajo, facilitarles como sea posible sus tareas, exigiendo siempre el respeto entre todos. Lo anterior son ventajas del trabajo en equipo, pero también existen algunas desventajas como puede ser la distracción o confusión de las tareas asignadas, el choque de personalidades, desilusiones por no llenar lo que uno considera que quiere hacer y conflicto en la equidad de responsabilidades, por lo que las metas pueden incumplirse. ${ }^{1,2}$

Uno de los mejores ejemplos del trabajo en equipo se ve en la labor diaria de los hospitales, ya que un gran número de las actividades que se llevan a cabo en una organización hospitalaria son gracias al trabajo en equipo, en donde la coordinación y la cooperación permiten el logro de objetivos. Los buenos hospitales se caracterizan porque todos sus elementos buscan el logro del objetivo: la atención cálida, segura y eficiente del paciente sin tener más protagonismo que el del éxito del objetivo, logrando que todos ganen, en especial el paciente, y por su puesto la organización hospitalaria. Pero iqué sucede cuando una de las partes del proceso falla? Todo el sistema se ve afectado y seguramente a pesar de que el resto del equipo haga su esfuerzo al máximo, el resultado no será el mismo como si todos hubieran funcionado de manera adecuada.

En la atención médica, el trabajo en equipo siempre busca conseguir el bienestar y la salud del paciente, en especial en la atención hospitalaria, pero además tiene un efecto potencializador en el equipo, pues los resultados son mejores que si cada uno de los participantes trabajara por separado y después sólo sumara cada quien su parte a la de los otros. El trabajo en equipo dentro del hospital permite que se conjunten habilidades, dones y talentos, haciendo que las tareas se completen; cuando se presentan problemas, es más fácil encontrar la solución al pertenecer a un equipo, y favorece la motivación entre los miembros, disminuyendo la rotación de personal y el desgaste laboral. ${ }^{3}$

Nadie dice que trabajar en equipo sea fácil, pero facilita el trabajo y mejora los resultados. Existen barreras para Ilevarlo a cabo, por ejemplo el tener multiempleo, pues puede evitar el desarrollo del sentimiento de pertenencia a

\footnotetext{
* Médico Internista. Coordinador de Pregrado y Postgrado, Hospital Angeles Health System. Catedrático de la Facultad Mexicana de Medicina de la Universidad La Salle, México.

* Coordinador del Departamento de Psiquiatría. Jefe de Postgrado de la Facultad Mexicana de Medicina de la Universidad La Salle, México.

$\S$ Director Médico.
}

Hospital Angeles Pedregal. México.

Correspondencia:

Federico L Rodríguez Weber

Correo electrónico: fweber@saludangeles.com

www.medigraphic.com/actamedica

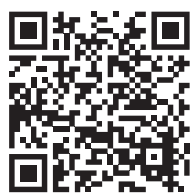



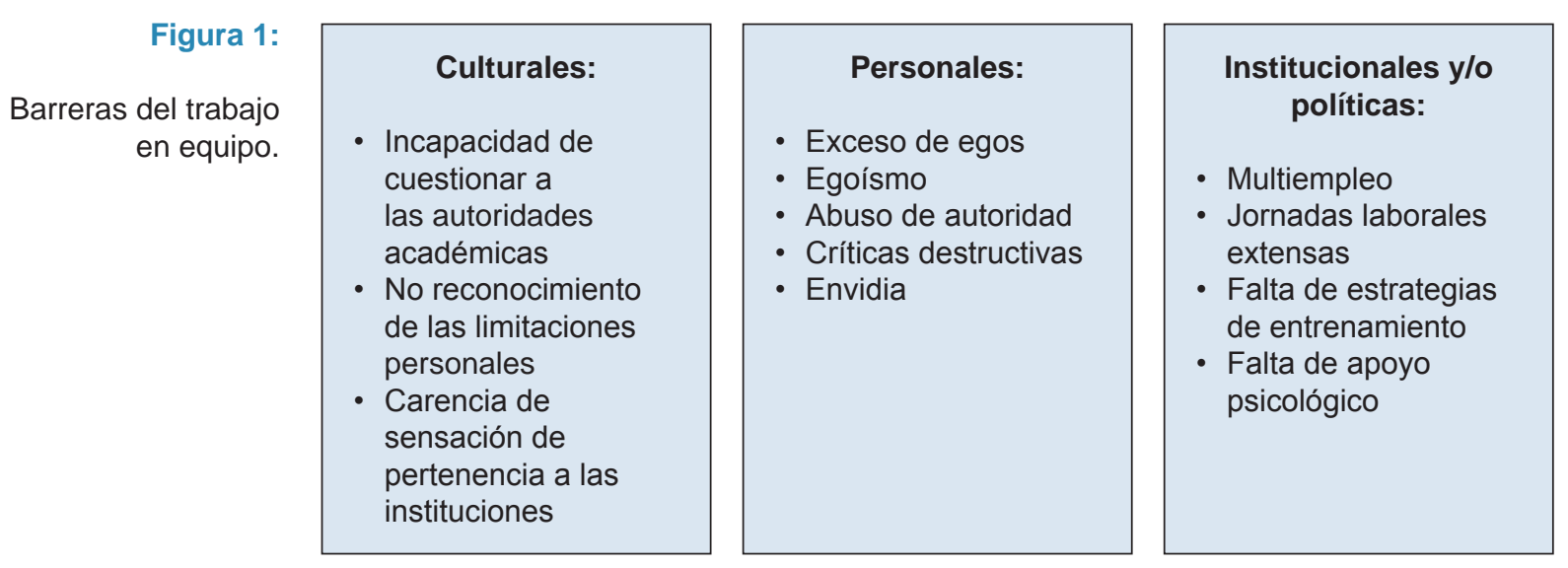

una institución (amor a la camiseta), la fatiga laboral es una de las grandes barreras para el éxito del trabajo en equipo, así como la minimización de la opinión de los miembros menos experimentados, fenómeno que no es raro en el ejercicio de la medicina, por ejemplo, en algunas residencias médicas, los de menor jerarquía no tienen derecho a opinar, afectando de esta manera el objetivo; otra situación perjudicial para el trabajo en equipo es cuando un médico con gran reconocimiento académico o jerarquía considera (y hace que consideren) que su opinión es valedera sólo por haberse originado en él, sin escuchar la opinión de los otros miembros.

A continuación, presentamos un cuadro con algunas de las barreras para el trabajo en equipo dentro del área de la salud, en especial en los hospitales (Figura 1). ${ }^{4}$

Recordemos que el trabajo en equipo no marca más que un pequeño engrane, que a su vez en algún punto se relaciona con otros sistemas, los cuales también se van a insertar en el macrosistema de trabajo. El éxito sólo se obtiene con el cumplimiento de cada uno de los integrantes del grupo de trabajo, lo cual permite que podamos dar una atención segura, eficiente y de calidad para nuestros pacientes. El binomio médico paciente se lleva en forma relativamente fácil en el consultorio al que el paciente acude por alguna enfermedad o con síntomas que ameriten ser evaluados para hacer un diagnóstico, pero la relación médico paciente en un hospital es muy diferente y aquí es donde el trabajo en equipo es esencial, además interviene un buen número de personas desde que ingresa por admisión o por urgencias, así como personas con diferentes conocimientos y responsabilidades, personal administrativo, de enfermería, de intendencia, técnicos de laboratorio y de radiología, ingenieros biomédicos, médicos en formación y médicos tratantes, todos deben trabajar en forma sincronizada, solo el trabajo en equipo permite que así sea, cualquier falla en una cadena de acciones puede afectar todo el proceso y, desde luego, la calidad de la atención médica, y puede ser muy grave que la seguridad del paciente se vea comprometida, este objetivo de sincronizar a todo el personal involucrado en la atención médica de un paciente hospitalizado requiere del liderazgo de alguien que conozca todos los pasos para otorgar una atención médica eficaz y segura. Esta función recae en el director del hospital, que debe ser de preferencia médico, pero con amplios conocimientos gerenciales. El objetivo de proporcionar la atención de calidad y seguridad es muy complejo, pero en forma simple se puede decir que requiere de manuales de organización de cada una de las áreas del hospital, de protocolos estandarizados y de líneas de acción para cumplir con los objetivos de cada departamento.

A manera de ejemplo de lo anterior, comento que un primer equipo puede ser el grupo administrativo que recibe al paciente, el cual lo pone en contacto con un segundo equipo, que es el de atención en piso, el cual entra en contacto con el personal administrativo en diferentes momentos (al recibir al paciente, al presentar al personal, al generar cargos, etcétera), éste dará la atención con los equipos de enfermería y médicos en los diferentes turnos y a su vez entrará en relación con otros equipos, como los de estudios de gabinete y laboratorio, que entran en contacto con el grupo de administrativos por otros caminos, los médicos entrarán en contacto con otros equipos si es necesario, por ejemplo con los de quirófano, quienes tendrán contacto con otros como los camilleros, intendencia, etcétera. El equipo directivo interactúa de alguna forma con cada uno de los equipos y con muchos grupos de trabajo en el hospital, como por ejemplo los terceros pagadores.

Todos los que conforman el personal de salud de una institución hospitalaria son personas con diferentes perfiles, conocimientos y destrezas en su oficio o en su profesión, pero todos trabajan en equipo en situaciones normales o de crisis permiten que la atención médica sea óptima y de calidad. 


\section{REFERENCIAS}

1. Arnold M, Osorio F. Introducción a los conceptos básicos de la teoría general de sistemas. Cinta moebio. 1998; 3: 40-49.

2. Fapohunda T. Towards effective team building in the workplace. Int J Educ Res. 2013; 1 (4): 1-12.
3. Erazo A. Un enfoque sistémico para comprender y mejorar los sistemas de salud. Rev Panam Salud Publica. 2015; 38 (3): 248253.

4. Rando Huluk AEK. Trabajo en equipo: ies posible formar equipos médicos expertos a partir de profesionales expertos? Rev Méd Urug. 2016; 32 (1): 59-67. 